\title{
Andrographolide inhibits NF-кB activation and attenuates neointimal hyperplasia in arterial restenosis
}

\author{
Yu-Jiu Wang, ${ }^{1, *}$, Jin-Tao Wang, ${ }^{2, *}$, Quan-Xin Fan ${ }^{1}$, Jian-Guo Geng ${ }^{2}$ \\ ${ }^{I}$ Department of Cardiac Surgery, Shandong Provincial Hospital, Shandong University, Jinan 250021, China; ${ }^{2}$ Laboratory of Molecular \\ Cell Biology, Institute of Biochemistry and Cell Biology, Shanghai Institutes for Biological Sciences, The Graduate School of Chinese \\ Academy of Sciences, Chinese Academy of Sciences, Shanghai 200031, China
}

The NF- $\kappa B$ transcription factors modulate the expression of tissue factor (TF), E-selectin (CD62E) and vascular cell adhesion molecule-1 (VCAM-1), which are essential for thrombosis and inflammation. We have previously shown that andrographolide (Andro) covalently modifies the reduced cysteine ${ }^{62}$ of p50 - a major subunit of NF- $\mathrm{kB}$ transcription factors, thus blocking the binding of NF- $\mathrm{KB}$ transcription factors to the promoters of their target genes, preventing NF$\kappa \mathrm{B}$ activation and inhibiting inflammation in vitro and in vivo. Here we report that Andro, but not its inactive structural analog $4 \mathrm{H}$-Andro, significantly suppressed the proliferation of arterial neointima ( $\sim 60 \%$ reduction) in a murine model of arterial restenosis. Consistently, $p 50^{-/}$mice manifested attenuated neointimal hyperplasia upon arterial ligation. Notably, the same dosage of Andro did not further reduce neointimal formation in $p 50^{-/-}$mice, which implicates the specificity of Andro on p50 for treating experimental arterial restenosis. The upregulation of NF- $\kappa \mathrm{B}$ target genes, including TF, Eselectin and VCAM-1, and the increased deposition of leukocytes (mainly CD $68^{+}$macrophages) were clearly detected within the injured arterial walls, all of which were significantly abolished by treatment with Andro or genetic deletion of p50. The expression of TF, E-selectin and VCAM-1 was also markedly upregulated in the patient sample of thrombotic vasculitis, indicating the clinical relevance of NF- $\mathrm{\kappa B}$ activation in the pathogeneses of occlusive arterial diseases. Our data thus indicate that, by the downregulation of the NF- $\mathrm{B}$ target genes that are critical in thrombosis and inflammation, specific inhibitors of $\mathrm{p} 50$, such as Andro, may be therapeutically valuable for preventing and treating thrombotic arterial diseases, including neointimal hyperplasia in arterial restenosis.

Keywords: NF-кB transcription factors, andrographolide, neointimal hyperplasia, arterial restenosis, TF, E-selectin, VCAM-1

Cell Research (2007) 17: 933-941. doi: 10.1038/cr.2007.89; published online 16 October 2007

\section{Introduction}

Atherosclerosis is a systemic arterial disease that originally involves the intima (affecting the media and adventitia to a less extent) of large- and medium-size arteries, including the coronary, cerebral, carotid, aortic and peripheral

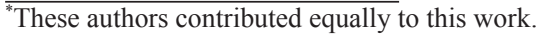
Correspondence: Jian-Guo Geng

Tel: 86-21-54921351

E-mail: genglab@gmail.com

Received 5 February 2007; revised 10 May 2007; accepted 8 July 2007; published online 16 October 2007
}

arteries. It progresses through the expansion of the lipid core and the accumulation and migration of macrophages at the edges of the plaque. The rupture and/or erosion of the fibrous cap triggers thrombus formation and arterial occlusion, leading to unstable angina, acute myocardial infarction, sudden cardiac death and stroke. Although the placement of a stent (a hollow cylinder that stretches open an arterial blockage) by percutaneous transluminal angioplasty has become the principal treatment for atherothrombosis, $15-20 \%$ patients (probably up to $30-60 \%$ patients with complex lesions) develop severe neointimal hyperplasia that overgrows the stent, causing arterial restenosis.

The NF- $\kappa$ B family of transcription factors regulates the 
expression of a wide range of genes. It has five members:

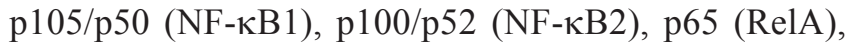
RelB and c-Rel. Among them, a dominant form is the $\mathrm{p} 50 /$ p65 heterodimer. The inactive NF- $\mathrm{kB}$ complex is localized in the plasma in the complex with the I $\kappa \mathrm{B}$ inhibitory factors. When activated, IкBs are rapidly phosphorylated and degraded to allow the shuttling of the p50/p 65 heterodimer into the nucleus $[1,2]$. The binding of $\mathrm{p} 50$ to the $\kappa \mathrm{B}$ motif causes transcription of multiple target genes, such as cell adhesion molecules (E-selectin and vascular cell adhesion molecule-1 (VCAM-1)), cytokines/chemokines, immunoreceptors, tissue factor (TF) and several cell-survival genes $[3,4]$. Numerous clinical investigations have suggested that $\mathrm{NF}-\mathrm{\kappa B}$ activation is involved in the host response to arterial injury [5-7] and may have an important role in neointimal hyperplasia in the mouse carotid ligation model [8].

In addition to the long history of using Andro as a remedy for inflammatory disorders, we have recently reported that andrographolide (Andro) covalently conjugates the reduced cysteine ${ }^{62}$ of $\mathrm{p} 50$, thus blocking NF- $\mathrm{KB}$ DNA binding and preventing NF- $\mathrm{BB}$-activated inflammation in the murine models of acute peritonitis, septic shock and allergic lung inflammation [9]. In this study, we sought to examine whether NF- $\mathrm{KB}$ activation has a critical role in the pathogenesis of arterial restenosis and whether Andro, by inhibition of NF- $\kappa \mathrm{B}$ activation, suppresses neointimal hyperplasia in experimental arterial restenosis.

\section{Materials and Methods}

\section{Animals}

Breeding pairs of $p 50^{-/}$mice (B6; 129P2-Nfik $1^{\text {tmlBal }} / \mathrm{J}$, p50 KO mice) and wild-type (WT) control mice (B6129PF2/J) were purchased from The Jackson Laboratory (Bar Harbor, ME, USA). C57 (C57BL/6J) mice were purchased from the Shanghai Animal Center of Chinese Academy of Sciences. They were housed in the Shanghai Animal Center of Chinese Academy of Sciences. The experimental protocol was approved by the Institutional Animal Care and Use Committee (IACUC) of the Chinese Academy of Sciences.

\section{Reagents}

Andro was purchased from Aldrich (Milwaukee, WI, USA), and $5 \mathrm{mg} / \mathrm{ml}(15 \mathrm{mM})$ stock was prepared by dissolving Andro in DMSO (Sigma-Aldrich, St Louis, MO, USA). 4-Hydro-andrographolide (4H-Andro) was prepared via the palladium on activated carboncatalyzed hydrogenation [9], to serve as the inactive structural analog of Andro as the negative control.

\section{Isolation of primary cells and cell culture}

The ethical review boards of the Chinese Academy of Sciences approved these studies. Informed consent was provided according to the Declaration of Helsinki. Human adherent monocytes were isolated from fresh human blood according to a previously published protocol [10]. Murine macrophages were obtained by peritoneal lavage of C57 mice, according to a previously published method
[11]. The purity of monocytes was routinely $>95 \%$ and viability was greater than $>96 \%$, measured through trypan blue exclusion. Human umbilical endothelial cells (HUVECs) and murine pulmonary vein endothelial cells (PVECs) were cultured as previously described $[8,12,13]$.

\section{Arterial injury model}

To induce arterial injury, male mice ( 7 weeks old) were anesthetized with an intraperitoneal injection of a solution of xylazine (12.5 $\mathrm{mg} / \mathrm{kg})$, ketamine $(125 \mathrm{mg} / \mathrm{kg})$ and atropine $(0.025 \mathrm{mg} / \mathrm{kg})$ diluted in an equal volume of saline ( $0.9 \%$ sodium chloride solution). Through a midline incision in the neck, the left common carotid artery was looped proximally to the carotid bifurcation and ligated completely by a 7-0 propylene suture to disrupt blood flow $[14,15]$. In the groups receiving treatment, Andro, $4 \mathrm{H}$-Andro ( $5 \mu \mathrm{g} / \mathrm{g}$ of body weight) or DMSO (in equal volume to the Andro group) was administered every second day beginning on day 0 . Animals were euthanized on day 28 with an overdose of pentobarbital $(210 \mathrm{mg} / \mathrm{kg}$ intraperitoneally). A 24-gauge catheter was then placed in the left ventricle for in situ perfusion of $4 \%$ paraformaldehyde in PBS for tissue fixation. Both injured left and uninjured right carotid arteries were excised, fixed and paraffin-embedded. Serial sections $(5 \mu \mathrm{m})$ at $1 \mathrm{~mm}$ below the ligated site through to the aortic arch were performed for histomorphometric examination and immunohistochemical staining.

\section{Histology}

The carotid artery was fixed by immersion in Bouin's fixation solution (75\% picric acid, $24 \%$ formaldehyde, $1 \%$ acetic acid) at $22{ }^{\circ} \mathrm{C}$ for $24 \mathrm{~h}$ followed by paraffin-embedding [16]. Serial sections of $5 \mu \mathrm{m}$ thick were cut at $1 \mathrm{~mm}$ below the ligated site through to the aortic arch. For morphometric analysis, the arterial sections were stained with elastica-van Gieson, and the intimal thickness was quantitatively analyzed using a BX50 microscope (Olympus, Tokyo, Japan) and NIH image (version 1.6) software package. The areas within the external elastic lamina (EEL area), the internal elastic lamina (IEL area) and the lumen were measured. The intimal area was determined by subtracting the lumen area from the area defined by IEL. The medial area was calculated by subtracting the IEL area from the EEL area. The ratios of the intimal:medial areas were also calculated. Morphometric analysis was performed in a double-blinded manner.

In addition, the arterial sections were stained with hematoxylin and eosin. Leukocytes on the endothelium and within the intima were counted double-blindly under a CX31 microscope (Olympus, Tokyo, Japan) at $\times 1000$ magnification. The percentage of leukocytes was calculated by dividing the number of counted leukocytes by the total number of cells within the adventitia [9].

\section{Immunohistochemical and immunofluorescence staining}

For immunohistochemical staining, the tissue sections were subsequently dewaxed and endogenous peroxidase was quenched with $3 \% \mathrm{H}_{2} \mathrm{O}_{2}$ in methanol. Prior to staining, non-specific binding was blocked by $10 \% \mathrm{BSA}$ in $50 \mathrm{mM}$ Tris-HCl, $\mathrm{pH} 7.6,0.15 \mathrm{M} \mathrm{NaCl}$ and $0.02 \% \mathrm{NaN}_{3}(\mathrm{TBS})$ at $37{ }^{\circ} \mathrm{C}$ for $30 \mathrm{~min}$. They were then incubated with rabbit preimmune $\operatorname{IgG}(8 \mu \mathrm{g} / \mathrm{ml})$, rabbit anti-mTF Ab $(1 \mu \mathrm{g} / \mathrm{ml})$, rabbit anti-E-selectin polyclonal $\mathrm{Ab}(6 \mu \mathrm{g} / \mathrm{ml})$ [16], anti-VCAM-1 polyclonal Ab (8 $\mu \mathrm{g} / \mathrm{ml}$; BD Pharmingen, San Diego, CA, USA) and rabbit anti-von Willebrand factor (vWF) polyclonal $\mathrm{Ab}(1: 500$ dilution; Sigma) in TBS containing $1 \%$ BSA at $4{ }^{\circ} \mathrm{C}$ overnight in a 
humidified chamber. The horseradish peroxidase-conjugated goat anti-rabbit $\operatorname{IgG~Ab}$ (Sigma) was applied at $37^{\circ} \mathrm{C}$ for $1 \mathrm{~h}$. After washing, color development was carried out by incubation with $0.05 \% 3-3^{\prime}$ diaminobenzidine (Sigma) in TBS supplemented with $0.1 \% \mathrm{H}_{2} \mathrm{O}_{2}$ for $10 \mathrm{~min}$. All incubations were followed by washing three times in TBS over $15 \mathrm{~min}$. Tissue sections were counterstained with hematoxylin and slides were mounted with coverslips $[9,16]$.

For immunofluorescent co-localization of TF, the tissue sections were treated with the same immunohistochemical steps as described above, and they were then incubated simultaneously with $10 \mu \mathrm{g} / \mathrm{ml}$ rabbit anti-mTF Ab, mouse anti-smooth muscle $\alpha$-actin monoclonal antibody (mAb; Sigma), $20 \mu \mathrm{g} / \mathrm{ml}$ rat anti-mouse CD68 mAb (Serotec) or $16 \mu \mathrm{g} / \mathrm{ml}$ biotinylated rabbit anti-human vWF Ab (Sigma).

\section{RNA isolation and quantitative RT-PCR}

Four days after ligation, approximately $20 \mathrm{mg}$ of arterial tissues were harvested for extracting total RNA using the RNA miniprep kit (Stratagene, La Jolla, CA, USA). Total RNA was eluted in $30 \mu 1$ RNase-free water and quantified using a BECMAN $640 \mathrm{M}$ spectrophotometer. Two micrograms of total RNA was reverse-transcribed into cDNA using M-MLV reverse transcriptase (Invitrogen, San Diego, CA, USA) in the presence of random primers. The cDNA was used to amplify the VCAM-1, E-selectin and TF fragments. To normalize RNA loading, the housekeeping gene $\beta$-actin was also amplified from each sample. The primer sequences for TF were as follows: sense 5'-GGA CAG CCA GTA ATT CAG CG-3', antisense 5'-GCT TGA GCC TTT CCG ATA AGT AA-3'. The primers for VCAM-1 (PPM03208B-200), E-selectin (PPM03195A-200) and $\beta$-actin (PPM02945A-200) were purchased from SuperArray Bioscience Corporation (Frederick, MD, USA). The RNA transcripts were quantified by an MX3000P real-time PCR (Stratagene), using Brilliant SYBR green qPCR master mix (SuperArray Bioscience Corporation) in $25-\mu 1$ reaction mixtures. Optimal primer concentrations were determined empirically.

\section{MTT assay}

Peritoneal macrophages from C57 mice, PVECs from B6 mice, HUVECs and human adherent monocytes $\left(5 \times 10^{3}\right.$ cells $\left./ \mathrm{ml}\right)$ were plated into 96 -well tissue culture plates. Five hours later, an aliquot of Andro ( $15 \mu \mathrm{M}$ final concentration) was added for $48 \mathrm{~h}$. An aliquot of $20 \mu \mathrm{l}$ methyl thiazolyl tetrazolium (MTT) was then added to each well. Four hours later, an aliquot of $100 \mu \mathrm{l}$ of $10 \% \mathrm{SDS} / 5 \%$ isobutanol $/ 0.12 \% \mathrm{HCl}$ was added to each well and the optical densities were measured at $\mathrm{OD}_{570 / 630 \mathrm{~nm}}[8]$.

\section{Statistical analysis}

All data were expressed as the mean \pm S.D. ANOVA and Student's unpaired $t$-test were used for statistical analysis. $P<0.05$ or 0.01 was considered statistically significant or very significant.

\section{Results}

Andro treatment and p50 ablation reduce neointimal hyperplasia

To investigate the functional role of Andro in neointimal proliferation during the pathogenesis of arterial restenosis, we administered Andro (a specific inhibitor for p50) and its inactive structural analog, $4 \mathrm{H}$-Andro, to the mice that underwent ligation of the left carotid arteries. Four weeks later, the ligated arteries were examined after euthanasia of these mice. Morphologically, the cross-sections of normal carotid arteries have irregular and thin vessel walls with large and wide internal diameters (Figure 1A, upper panel). By contrast, cross-sections of injured carotid arteries reveal round and thick vessel walls (which are due mainly to neointimal proliferation) with small and narrow internal diameters. Compared with those treated with $4 \mathrm{H}$-Andro and DMSO, Andro dramatically attenuated the proliferation of neointima and prevented the thickening of vessel walls. Ligation of the left carotid arteries triggered a profound proliferation of neointima, as demonstrated by an increased intimal area $\left[19 \pm 16\left(\times 10^{3} \mu \mathrm{m}^{2}\right)\right.$; Figure 1B] and a higher intimal:medial area ratio (I/M: $0.805 \pm 0.329$; Figure 1C). Treatment with Andro significantly reduced the growth of the neointima [intimal area: $8 \pm 6\left(\times 10^{3} \mu \mathrm{m}^{2}\right)$; I/M: 0.311 \pm 0.171 ; Figure $1 \mathrm{~B}]$, whereas treatment with $4 \mathrm{H}$-Andro [intimal area: $19 \pm 9\left(\times 10^{3} \mu \mathrm{m}^{2}\right)$; I/M: $0.808 \pm 0.417$; Figure $1 \mathrm{~B}$ ] and DMSO [intimal area: $20 \pm 9\left(\times 10^{3} \mu \mathrm{m}^{2}\right)$; I/M: 0.78 \pm 0.301 ; Figure $1 \mathrm{~B}]$ did not. By contrast, the medial areas remained unchanged among all groups.

Consistent with the above observations, we found that the neointimal hyperplasia was reduced in $p 50^{-/-}$mice (p50 $\mathrm{KO}$ ) following ligation of the left carotid artery (Figure $1 \mathrm{~A}$, lower panels). The mean intimal area was $12 \pm 3\left(\times 10^{3}\right.$ $\left.\mu \mathrm{m}^{2}\right)$ in $p 50^{--}$mice compared with $30 \pm 15\left(\times 10^{3} \mu \mathrm{m}^{2}\right)$ in B6 mice (Figure 1D). p50 KO mice manifested a smaller $\mathrm{I} / \mathrm{M}$ ratio $(0.434 \pm 0.108)$ when compared with $\mathrm{B} 6$ mice $(1.080 \pm 0.393$; Figure 1E). There was also no significant difference in medial areas among all groups. Importantly, the thickness of the neointima (Figure 1D and 1E) was not further reduced by treatment with an identical dosage of Andro in $p 50^{-/}$mice, suggesting the specificity of Andro on p50 for reducing neointimal hyperplasia in the murine model of arterial restenosis.

\section{Andro treatment and p50 knockout suppress the expression of E-selectin, VCAM-1 and TF}

The de novo synthesis of E-selectin and VCAM-1 in vascular endothelial cells is well known to be exclusively regulated by $\mathrm{NF}-\kappa \mathrm{B}$ transcription factors $[9,14-20]$. We thus examined whether the expression of E-selectin and VCAM-1 is regulated by $\mathrm{p} 50$-mediated NF- $\mathrm{\kappa B}$ activation in our murine models of experimental arterial restenosis. As expected, the expression of E-selectin and VCAM-1 was undetectable on the endothelial layers of normal carotid arteries (Figure 2A). However, they were markedly upregulated in the arterial walls of the model groups. Compared with the model groups and the $4 \mathrm{H}$-Andro groups, treatment with Andro significantly reduced the staining of E-selectin and VCAM-1 in the arterial walls. Consistently, 
A



Sham

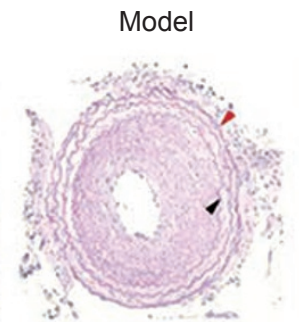

B6



p50 KO
4H-Andro

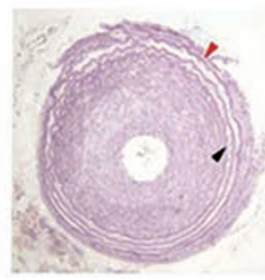

p50 KO + Andro



$\therefore$.

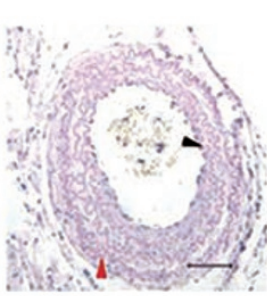

B

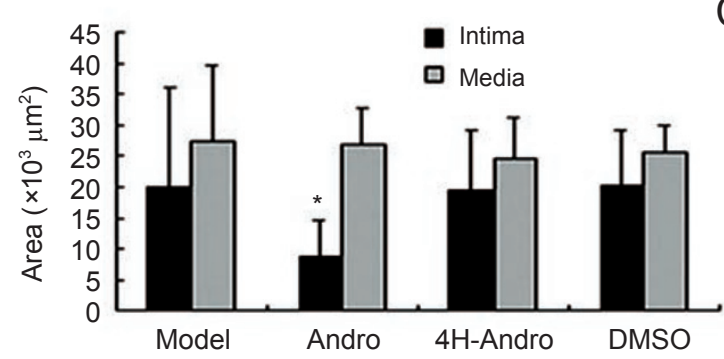

C

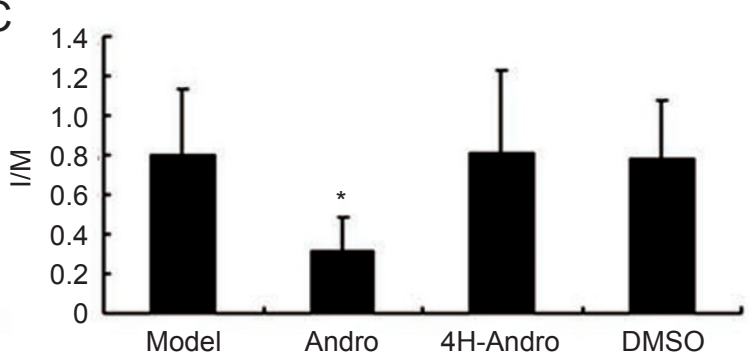

E

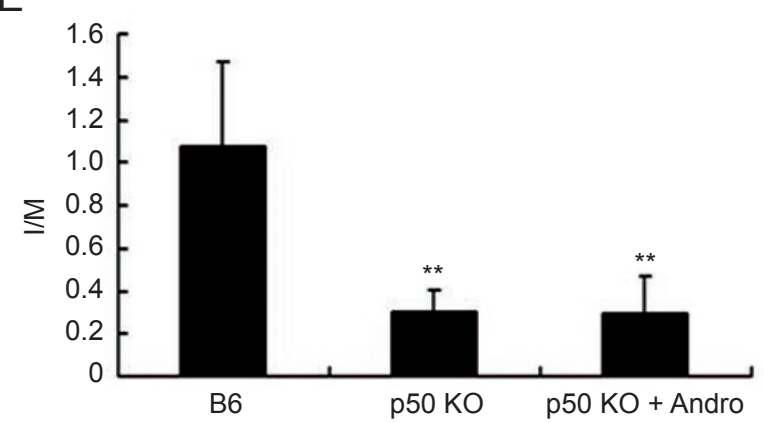

Figure 1 Inhibition of p50 attenuates arterial restenosis. (A) Cross-sections of carotid arteries stained with EVG following ligation of the carotid artery for 4 weeks. Red arrow heads point to the external elastic lamina, and black arrow heads point to the internal elastic lamina in C57 mice (upper panel) and B6 and p50 KO (lower panel). (B, C) Andro inhibits neointimal hyperplasia. C57 mice were either untreated (model group, $n=10$ ) or treated with Andro (Andro group, $n=9$ ), 4H-Andro (4H-Andro group, $n=8$ ) or DMSO (DMSO group, $n=8)$. The left carotid arteries were ligated while the right carotid arteries were untouched as a negative control. (D, E) p50 deficiency abrogates neointimal hyperplasia. Following ligation of the left carotid artery, B6 mice $(n=9)$ and p50 KO mice (untreated, $n=8$ or treated, $n=7$ ) were either untreated or treated with Andro. Results are presented as the mean \pm S.D. from two separate experiments. ${ }^{*} p<0.05$ and ${ }^{*} * P<0.01$ compared with the corresponding model groups. The scale bar indicates $50 \mu \mathrm{m}$.

the expression of E-selectin and VCAM-1 was almost completely absent in the model groups of $\mathrm{p} 50 \mathrm{KO}$ mice (Figure 2B). As a consequence of inhibiting the p50-mediated expression of leukocyte adhesion molecules, such as E-selectin and VCAM-1, the deposition of leukocytes, mainly macrophages and/or neutrophils, in the intima of arterial walls was dramatically decreased in Andro-treated mice (Figure 2D) and p50 KO mice (Figure 2F) compared with their respective model groups.

As the p50/65 heterodimer, the dominant complex of 
$\mathrm{NF}-\kappa \mathrm{B}$ transcriptional factors, reportedly regulates $\mathrm{TF}$ at the transcriptional level [21-24], we next examined TF expression in our in vivo model of arterial restenosis. Compared with the sham groups, TF expression was markedly upregulated in the model groups of arterial restenosis (Figure 3A, upper panel and Figure 3B). In contrast to 4HAndro, treatment with Andro significantly suppressed TF expression. Consistently, TF expression was also reduced in the model groups of $550 \mathrm{KO}$ mice compared with those in the model groups of B6 mice (Figure 3A, lower panel and Figure 3C).

In searching for the cellular distribution of $\mathrm{TF}$, we car- ried out an immunofluorescence co-localization study using laser scanning confocal microscopy. TF expression was co-localized with CD68 (a marker for macrophages; Figure 4, upper panel), vWF(a marker for endothelial cells; Figure 4 , middle panel) and $\alpha$-actin (a marker for smooth muscle cells; Figure 4, lower panel). These findings demonstrate that TF overexpression in infiltrated macrophages stimulated endothelial cells and injured vascular smooth muscle cells in our experimental model of arterial restenosis.

To determine the mRNA expression of E-selectin, VCAM-1 and TF, total RNA was extracted from the carotid artery specimen 4 days after ligation of the left
A
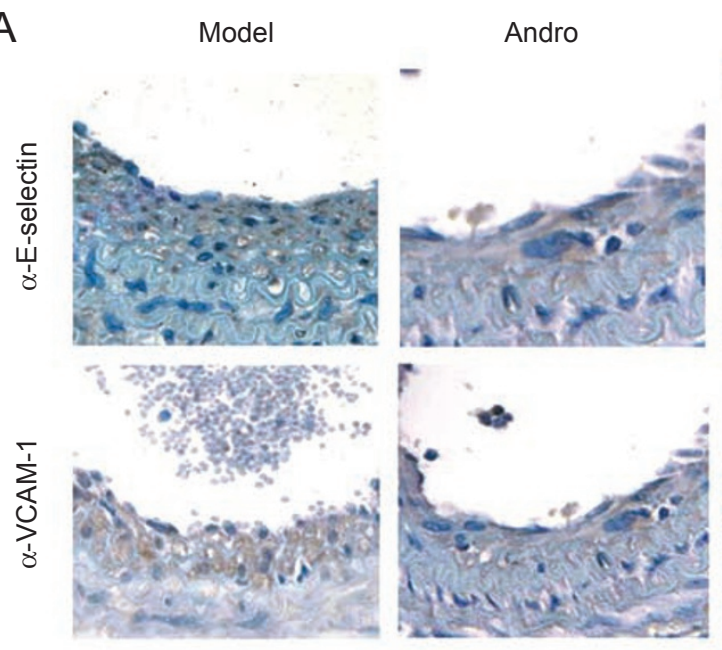

B

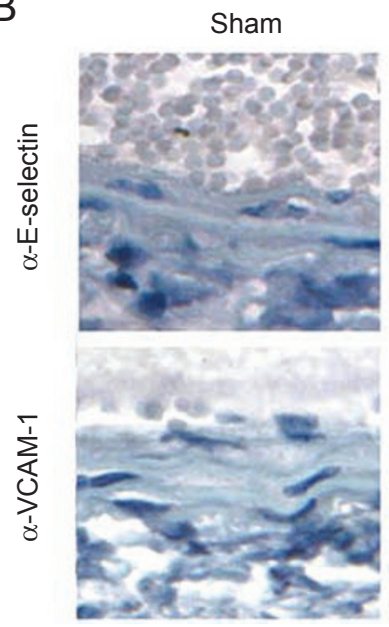

B6

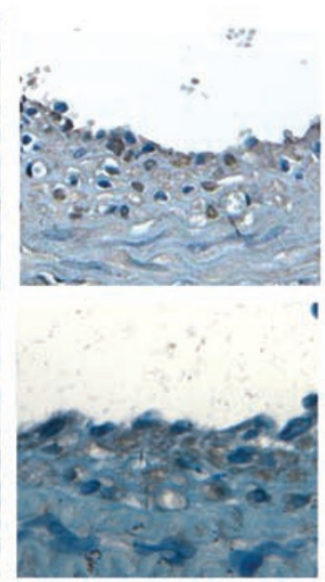

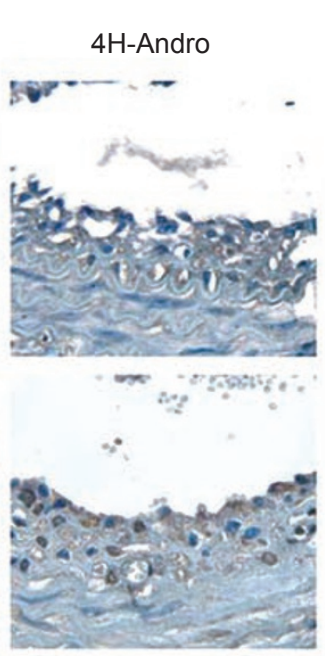

p50 KO

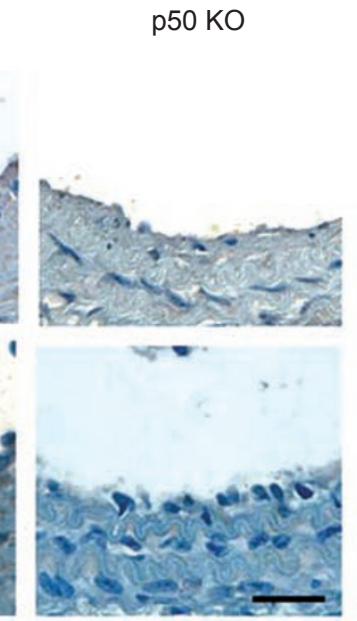

C
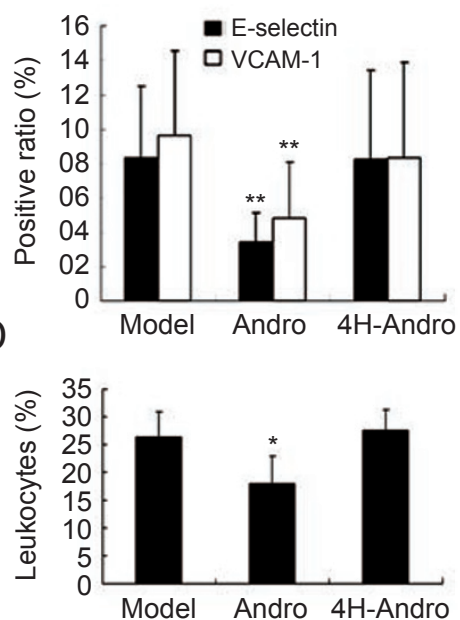

$E$

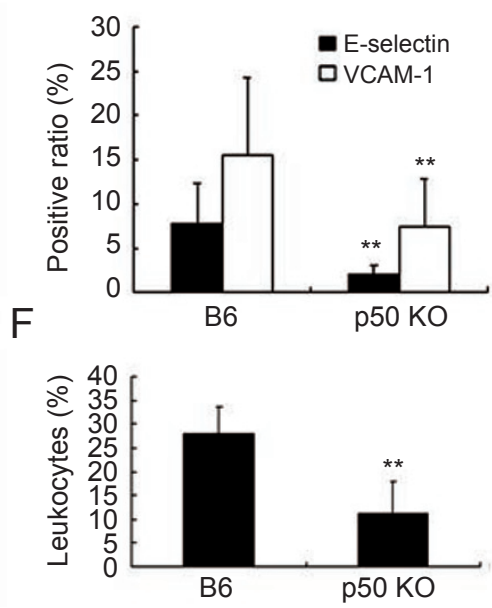

Figure 2 Andro treatment and p50 deficiency downregulate the expression of E-selectin and VCAM-1 and suppress leukocyte infiltration in injured arteries. (A-C, E). Immunohistochemical staining and gray-scale analysis of E-selectin and VCAM-1 in the cross-sections of carotid arteries from C57 mice $(\mathbf{A}, \mathbf{C})$ and $\mathrm{B} 6$ and $p 50 \mathrm{KO}$ mice $(\mathbf{B}, \mathbf{E})$ were performed as described before [9]. (D, F) Leukocyte depositions in the neointima of injured carotid arteries. Results displayed are representative of multiple sections of more than five samples from at least two separate experiments, which are presented as the mean \pm S.D. of five respective fields for each section (three sections per animal) examined. ${ }^{*} p<0.05$ and ${ }^{*} p p<0.01$ compared with the corresponding model groups. The scale bar indicates $50 \mu \mathrm{m}$. 
A

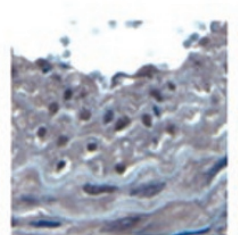

Model



Sham

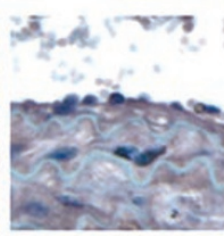

Andro

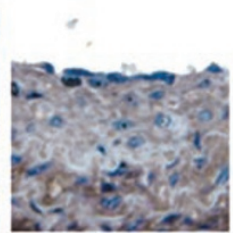

B6



$4 \mathrm{H}-\mathrm{Andro}$

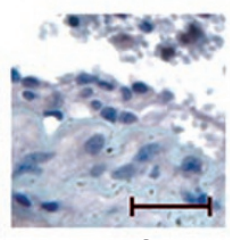

p50 KO
$\mathrm{B}$



C

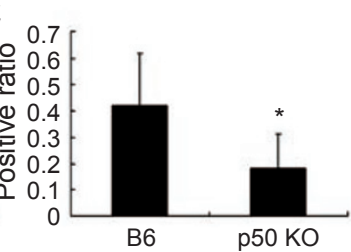

Figure 3 Inhibition of p50 downregulates TF. (A) TF immunohistochemical staining of the uninjured (sham) and injured arteries. (B, C) A gray-scale analysis of TF immunohistochemical staining. Results are presented as either a representative of three independent experiments or the mean \pm S.D. of triplicate measurements from two independent experiments. ${ }^{*} p<0.05$ compared with the corresponding model group. The scale bar indicates $25 \mu \mathrm{m}$.

carotid artery. Indeed, the mRNA expression of E-selectin, VCAM-1 (Figure 5A and 5B) and TF (Figure 5C) was markedly elevated in the ligated artery. Compared with the mice treated with $4 \mathrm{H}$-Andro, the mice treated with Andro had a significantly reduced expression of mRNAs of Eselectin, VCAM-1 and TF in the injured left carotid artery.

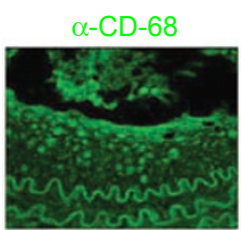

$\alpha-v W R$

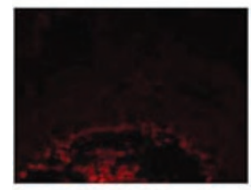

$\alpha-\alpha-$-Actin

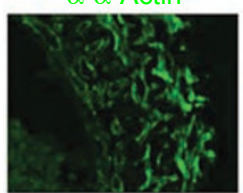

$\alpha-\mathrm{TF}$



$\alpha-\mathrm{TF}$



$\alpha-T F$

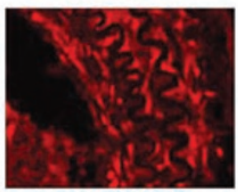

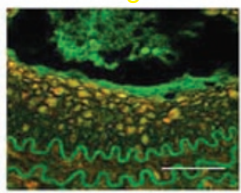
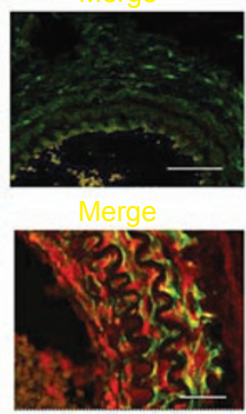

Figure 4 Cellular distribution of TF in experimental arterial restenosis. Arterial sections were stained for TF, which was completely co-localized with infiltrated macrophages (CD68 positive; upper panel), activated endothelial cells (vWF positive; middle panel) and partially co-localized with stimulated smooth muscle cells ( $\alpha$-actin positive; low panel). Results are representative of three independent experiments. The scale bars indicate $30 \mu \mathrm{m}$ in the upper panel, 50 $\mu \mathrm{m}$ in the middle panel and $20 \mu \mathrm{m}$ in the lower panel.

Our data thus argue for the exclusive regulation of these $\mathrm{NF}-\kappa \mathrm{B}$ target genes by $\mathrm{p} 50$ in our models of experimental arterial restenosis.

Overexpression of $N F-\kappa B$ target genes in thrombotic vasculitis

To correlate our experimental results in mice with the pathological changes in patients with occlusive arterial diseases, we obtained a human sample of thrombotic vasculitis and examined the expression of various NF- $\kappa B$ target


Figure 5 Treatment with Andro downregulates mRNA expression of NF- $\kappa$ B target genes. The mRNAs for VCAM-1 and E-selectin (A) and TF (B) in the carotid arteries were determined using quantitative RT-PCR. Results are presented as the mean \pm S.D. of triplicate measurements from two independent experiments. $* p<0.05$ compared with the corresponding model groups. 



Figure 6 Expression of E-selectin, VCAM-1 and TF in thrombotic vasculitis. The human tissue sections of thrombotic vasculitis were stained with rabbit preimmune rabbit IgG or Abs to E-selectin, VCAM-1 and TF. The scale bar indicates $25 \mu \mathrm{m}$.

genes, such as E-selectin, VCAM-1 and TF. We found that E-selectin, VCAM-1 and TF were highly expressed in the pathological specimen of thrombotic vasculitis (Figure 6), supporting the clinical relevance of our present findings in experimental arterial restenosis.

\section{Andro fails to inhibit cell growth}

We further tested whether Andro had any detectable effect on the growth of HUVECs, human adherent monocytes, peritoneal macrophages from $\mathrm{C} 57$ mice and PVECs from B6 mice. Using the MTT assay, we found that compared with the sham-treated cells, $15 \mu \mathrm{M}$ Andro (the identical concentration used in this study in vivo) did not affect the growth of these primary cells in vitro (Figure 7), ruling out the possible cytotoxic action of Andro for the prevention of neointimal hyperplasia in our murine model of arterial restenosis.

\section{Discussion}

In this study, we have revisited the functional importance of NF- $\mathrm{KB}$ activation in the pathogenesis of arterial restenosis, using two divergent approaches - pharmacological treatment with Andro (an inhibitor of p50) and genetic deletion of $p 50$. Consistent with previous findings $[5,8$,
25-29] in which the activation of NF- $\kappa$ B transcription factors, especially $p 50$, is critical to neointimal hyperplasia, we have found that treatment with Andro and $p 50$ ablation both significantly reduce neointimal formation without affecting medial thickening, attesting to the significance of $\mathrm{NF}-\kappa \mathrm{B}$ activation in the pathogenesis of arterial restenosis. Importantly, these experimental findings in mice are supported by the expression profiles of E-selectin, VCAM-1 and $\mathrm{TF}$ in samples of human thrombotic vasculitis.

$\mathrm{NF}-\kappa \mathrm{B}$ transcription factors regulate the expression of E-selectin and VCAM-1, which are cell adhesion molecules for leukocyte adhesion to stimulated endothelium. Upon arterial ligation, we found that E-selectin and VCAM-1 are upregulated, leading to the deposition of leukocytes, mainly $\mathrm{CD} 68^{+}$macrophages, in the intima of arterial walls (Figure $2 \mathrm{~A}$ and $2 \mathrm{~B}$ ). In contrast to $4 \mathrm{H}$-Andro and WT mice, treatment with Andro and $p 50$ deficiency significantly suppress the expression of E-selectin and VCAM-1 and consequently inhibit the leukocyte deposition that is presumably mediated by these leukocyte adhesion molecules, suggesting the pathological roles of E-selectin- and VCAM-1-induced leukocyte adhesion in neointimal hyperplasia.

$\mathrm{TF}$ is a $47-\mathrm{kDa}$ transmembrane glycoprotein, which serves as a principal initiator of blood clotting and a major trigger of arterial and venous thrombosis. TF forms the cell-surface complex with coagulation factor VII/VIIa and assembles the extrinsic tenase complex by generating coagulation factor $\mathrm{Xa}$, which consequently activates the prothrombinase complex for the formation of thrombin, thus initiating the coagulation cascade and leading to blood

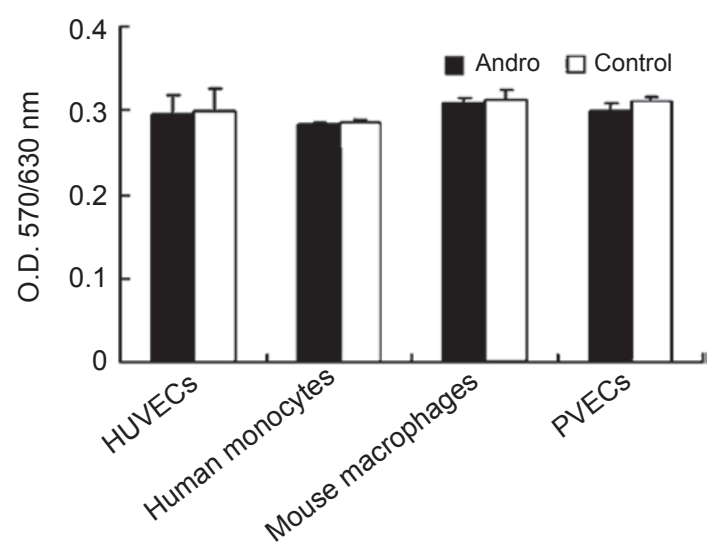

Figure 7 Testing of Andro cytotoxicity. HUVECs, human adherent monocytes, peritoneal macrophages from C57 mice and PVECs from B6 mice were incubated with $15 \mu \mathrm{M}$ Andro and their growth rates were measured using an MTT assay. Results presented are the mean \pm S.D. of the triplicate measurements from three independent experiments. 
clotting responsible for preventing excessive bleeding physiologically and causing arterial and venous thrombosis pathologically.

The expression of the TF gene is regulated principally at the transcriptional level [30]. Although resting and quiescent monocytes and endothelial cells express little or no TF constitutively, the level of this procoagulant increases dramatically in stimulated monocytes and vascular endothelial cells during the pathogeneses of various diseases, such as thromboembolism and atherosclerosis. In this context, we found that TF is upregulated on activated monocytes, endothelial cells and vascular smooth muscle cells (Figures 4 and 5), suggesting that it is critically involved in the pathogenesis of arterial restenosis.

Taken together, we propose that NF- $\kappa \mathrm{B}$ transcription factors critically regulate the expression of E-selectin, VCAM-1 and TF, which synergistically exaggerate inflammatory and thrombotic responses, during the pathogenesis of neointimal hyperplasia in the murine model of experimental arterial restenosis and human thrombotic vasculitis. As an inhibitor for NF- $\kappa \mathrm{B}$ activation, Andro is not only specific for p50 but also equally as potent as the genetic ablation of $p 50$, which suggests the therapeutic potential of $\mathrm{NF}-\kappa \mathrm{B}$ antagonists, such as Andro, in the prevention and treatment of occlusive arterial diseases including arterial restenosis.

\section{Acknowledgments}

This work was supported by grants from the National Natural Science Foundation of China (30370694, 30421005, 30623003, 30400245 and 30630036), the Ministry of Science and Technology of China (2002CB513006, 2006CB943902 and 2006AA02Z169), the Chinese Academy of Sciences (KSCX2-YW-R-67 and KJCX2-YW-H08) and the Shanghai Municipal Commission for Science and Technology (04JC14078, 06DZ22032, 055407035 and 058014578).

\section{References}

1 Schmitz ML, Mattioli I, Buss H, Kracht M. NF- $\kappa$ B: a multifaceted transcription factor regulated at several levels. Chembiochem 2004; 5:1348-1358.

2 Li Q, Verma IM. NF-kappaB regulation in the immune system. Nat Rev Immunol 2002; 2:725-734.

3 Lawrence T, Gilroy DW, Colville-Nash PR, Willoughby DA. Possible new role for NF-kappaB in the resolution of inflammation. Nat Med 2001; 7:1291-1297.

4 Yamamoto Y, Gaynor RB. Therapeutic potential of inhibition of the NF-kappaB pathway in the treatment of inflammation and cancer. J Clin Invest 2001; 107:135-142.

5 Cercek B, Yamashita M, Dimayuga P, et al. Nuclear factor-kap$\mathrm{paB}$ activity and arterial response to balloon injury. Atheroscle- rosis 1997; 131:59-66.

6 Lindner V. The NF-kappaB and IkappaB system in injured arteries. Pathobiology 1998; 66:311-320.

7 Landry DB, Couper LL, Bryant SR, Lindner V. Activation of the NF-kappa B and Ikappa B system in smooth muscle cells after rat arterial injury. Induction of vascular cell adhesion molecule1 and monocyte chemoattractant protein-1. Am J Pathol 1997; 151:1085-1095.

8 Squadrito F, Deodato B, Bova A, et al. Crucial role of nuclear factor-kappaB in neointimal hyperplasia of the mouse carotid artery after interruption of blood flow. Atherosclerosis 2003; 166:233-242.

9 Xia YF, Ye BQ, Li YD, et al. Andrographolide attenuates inflammation by inhibition of NF-kappa B activation through covalent modification of reduced cysteine 62 of p50. J Immunol 2004; 173:4207-4217.

10 Geng JG, Bevilacqua MP, Moore KL, et al. Rapid neutrophil adhesion to activated endothelium mediated by GMP-140. Nature 1990; 343:757-760.

11 Mercurio AM, Schwarting GA, Robbins PW. Glycolipids of the mouse peritoneal macrophage. Alterations in amount and surface exposure of specific glycolipid species occur in response to inflammation and tumoricidal activation. J Exp Med 1984; 160:1114-1125.

12 Marelli-Berg FM, Peek E, Lidington EA, et al. Isolation of endothelial cells from murine tissue. J Immunol Methods 2000; 244:205-215.

13 Ozcan M, Morton CT, Solovey A, et al. Whole blood tissue factor procoagulant activity remains detectable during severe aplasia following bone marrow and peripheral blood stem cell transplantation. Thromb Haemost 2001; 85:250-255.

14 Kumar A, Lindner V. Remodeling with neointima formation in the mouse carotid artery after cessation of blood flow. Arterioscler Thromb Vasc Biol 1997; 17:2238-2244.

15 Sirsjo A, Lofving A, Hansson GK, Wagsater D, Tokuno S, Valen G. Deficiency of nitric oxide synthase 2 results in increased neointima formation in a mouse model of vascular injury. $\mathrm{J}$ Cardiovasc Pharmacol 2003; 41:897-902.

16 Wang B, Xiao Y, Ding BB, et al. Induction of tumor angiogenesis by Slit-Robo signaling and inhibition of cancer growth by blocking Robo activity. Cancer Cell 2003; 4:19-29.

17 Geng JG, Raub TJ, Baker CA, et al. Expression of a P-selectin ligand in zona pellucida of porcine oocytes and P-selectin on acrosomal membrane of porcine sperm cells. Potential implications for their involvement in sperm-egg interactions. J Cell Biol 1997; 137:743-754.

18 Neish AS, Williams AJ, Palmer HJ, Whitley MZ, Collins T. Functional analysis of the human vascular adhesion molecule 1 promoter. J Exp Med 1992; 176:1583-1593.

19 Whitley MZ, Thanos D, Read MA, Maniatis T, Collins T. A striking similarity in the organization of the E-selectin and beta interferon gene promoters. Mol Cell Biol 1994; 14:64646475.

20 Shu HB, Agranoff AB, Nabel EG, et al. Differential regulation of vascular cell adhesion molecule 1 gene expression by specific NF- $\kappa \mathrm{B}$ subunits in endothelial and epithelial cells. Mol Cell Biol 1993; 13:6283-6289.

21 Tilley R, Mackman N. Tissue factor in hemostasis and thrombosis. Semin Thromb Hemost 2006; 32:5-10. 
22 Del Conde I, Shrimpton CN, Thiagarajan P, Lopez JA. Tissuefactor-bearing microvesicles arise from lipid rafts and fuse with activated platelets to initiate coagulation. Blood 2005; 106:16041611.

23 Morrissey JH. Tissue factor: in at the start...and the finish? J Thromb Haemost 2003; 1:878-880.

24 Polgar J, Matuskova J, Wagner DD. The P-selectin, tissue factor, coagulation triad. J Thromb Haemost 2005; 3:1590-1596.

$25 \mathrm{Bu}$ DX, Erl W, de Martin R, Hansson GK, Yan ZQ. IKKbetadependent NF-kappaB pathway controls vascular inflammation and intimal hyperplasia. FASEB J 2005; 19:1293-1295.

26 Inagaki H, Suzuki J, Ogawa M, Taniyama Y, Morishita R, Isobe M. Ultrasound-microbubble-mediated NF-kappaB decoy transfection attenuates neointimal formation after arterial injury in mice. J Vasc Res 2006; 43:12-18.

27 Kalinowski M, Viehofer K, Hamann C, et al. Local administration of NF-kappa B decoy oligonucleotides to prevent restenosis after balloon angioplasty: an experimental study in New Zealand white rabbits. Cardiovasc Interven Radiol 2005; 28:331-337.

28 Miyake T, Aoki M, Shiraya S, et al. Inhibitory effects of NF-kappaB decoy oligodeoxynucleotides on neointimal hyperplasia in a rabbit vein graft model. J Mol Cell Cardiol 2006; 41:431-440.

29 Ouchida R, Kusuhara M, Shimizu N, et al. Suppression of NF-kappaB-dependent gene expression by a hexamethylene bisacetamide-inducible protein HEXIM1 in human vascular smooth muscle cells. Genes Cells 2003; 8:95-107.

30 Mackman N. Regulation of the tissue factor gene. Thromb Haemost 1997; 78:747-754. 